\title{
Measurement of the Magnetic Field Distribution in Railguns Using CMR-B-Scalar Sensors
}

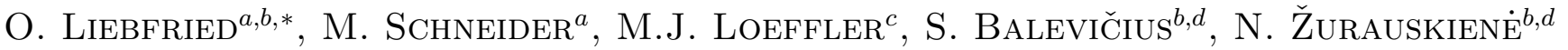 \\ AND V. STANKEVIČ $\check{\check{C}}^{b, d}$ \\ ${ }^{a}$ French-German Research Institute \\ 5 rue du Général Cassagnou, F-68301 Saint-Louis, France \\ ${ }^{b}$ Semiconductor Physics Institute, A. Goštauto 11, Vilnius, Lithuania \\ ${ }^{c}$ Gelsenkirchen University of Applied Sciences \\ Neidenburger Str. 10, D-45877 Gelsenkirchen, Germany \\ ${ }^{d}$ Vilnius Gediminas Technical University, Saulètekio 11, Vilnius, Lithuania
}

\begin{abstract}
Colossal magnetoresistance effect $B$-scalar magnetic field sensors with effective areas of $0.05 \mathrm{~mm}^{2}$ were used very close to the rails for magnetic field measurements. These measurements were performed during static and dynamic railgun experiments. In static experiments three different rail materials were used and the results are compared to a finite element simulation.
\end{abstract}

PACS numbers: 75.47.Gk, 07.55.Ge, 85.70.Rp

\section{Introduction}

One of the key points in understanding railgun behaviour is the current distribution in the rails. The current is strongly influenced by the fast moving projectile. Excessive current concentration near the contact interfaces leads to heating and wear-out effects of both, solid armatures and rails. If the magnetic field distribution around the conductors is known, conclusions concerning the current distribution can be drawn by using the BiotSavart law. Precise measurements not only improve the understanding of the experiment but also serve as a validation for difficult "multi-physics" numerical analysis.

To enable a precise measurement, magnetic field $(B$-field) sensors with small measurement volumes are needed. Furthermore, currently used high $B$-field sensors $(>1 \mathrm{~T})$ such as loop, Hall, magneto-optical or giant magnetoresistive sensors do not fulfill all the conditions like small size, accuracy or the usability under rough conditions (plasma, soot etc.) at the same time.

A new kind of sensor, consisting of thin manganite films exhibiting the colossal magnetoresistance effect (CMR), is able to measure $B$-fields up to $40 \mathrm{~T}$ under electromagnetic launch conditions [1]. A new generation of CMR-B-Scalar sensors, used for the experiments presented in this paper, has a very small volume and is therefore suitable to measure the $B$-field distribution close to the rails. An earlier paper [2] reported about the use

* corresponding author; e-mail: oliver.liebfried@isl.eu of these sensors in measuring magnetic field diffusion in railgun equipped with different material. Different rail material influences the current distributions due to the interplay between conductivity and skin effect. Here additional results are reported.

\section{Measurement setup}

The CMR- $B$-scalar sensor used for measurements is based on a thin $\mathrm{La}-\mathrm{Sr}-\mathrm{MnO}_{3}$ film. It has active area about $500 \times 50 \mu \mathrm{m}^{2}$ resulting in a very small volume. The distance between the measurement volume and the sensor tip was $400 \mu \mathrm{m}$. This allowed us $B$-field measurements very close to the rails. The details of the sensor design are described in [1].

Static railgun experiments were performed by short-circuiting the 1370-mm-long EMA3-type railgun [3]. It consisted of copper-chrome rails with a conductivity $\sigma=5 \times 10^{7} \mathrm{~S} / \mathrm{m}$ and a mechanical setup made from steel and glass fibre reinforced plastics (GRP). A cross-section of the rails together with the sensors' positions ( $\mathrm{P} 1-\mathrm{P} 5)$ is shown in Fig. 1. Five CMR-sensors are mounted in a plastic block positioned close to the rails. An insulating tape (thickness $=60 \mu \mathrm{m}$ ) separates the sensors and the rail. The distance $\Delta x$ between the rail and the sensors active area is varied during different experiments. Measurements are compared to simulations performed with the 3D finite element method (FEM) code MEGA [4]. The simulated current distribution in the rails at $I=140 \mathrm{kA}$, $t=0.2 \mathrm{~ms}$ is shown in Fig. 1 . 


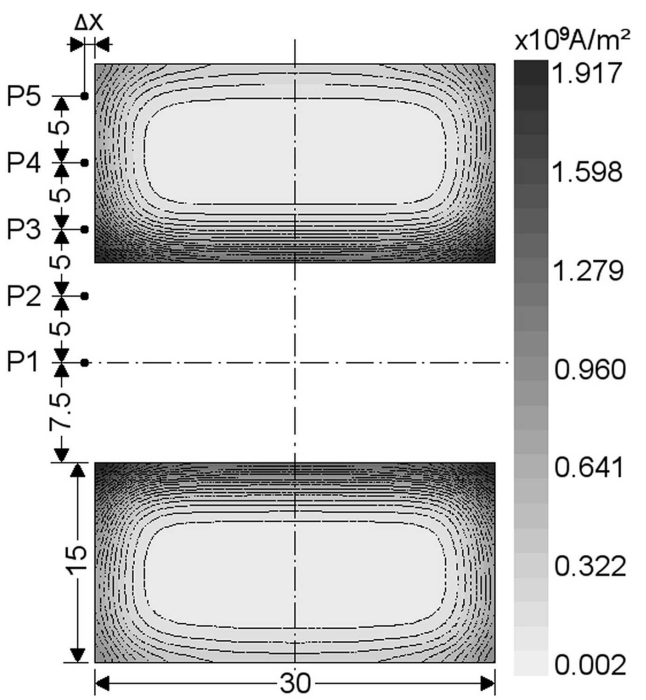

Fig. 1. EMA3 cross-section with sensor positions and simulated current distribution during a static experiment.

Dynamic railgun experiments were realized using the railgun RAFIRA [5]. The sensors are placed in a GRP block to protect them from electrical, mechanical and thermal damage. The thickness of the protective GRP layer between the sensors and the rails was $0.5 \mathrm{~mm}$ resulting in a total distance of about $1 \mathrm{~mm}$ between the sensor's active volume and the rails. The block was placed in a distance of $360 \mathrm{~mm}$ to the muzzle. The spacing of adjacent sensors was $5 \mathrm{~mm}$ in the horizontal plane and $3.12 \mathrm{~mm}$ in the vertical (see the inset in Fig. 4).

\section{Results and discussion}

A typical measurement of a magnetic field pulse during a static experiment with copper rails is shown in Fig. 2. The magnetic field measurement error (light gray area) was below $10 \%$ at fields stronger than $1.5 \mathrm{~T}$. At weaker $B$-fields the error increases to $20 \%$ due to a low field anisotropy of the CMR-effect [6]. The simulation (dashed line in Fig. 2) shows very good agreement with the measurement for $B>1.5 \mathrm{~T}$.

Figure 3 shows the $B$-field at $t=250 \mu \mathrm{s}$ as a function of the sensors' position (given in the insets). The simulation is compared to the measurement at three different distances $\Delta x$ between the rail and the sensors. The error bars display an error of $\pm 10 \%$. Measurement and simulation show the high current concentration at the inner rail edge $(y=7.5 \mathrm{~mm})$ due to the proximity effect. The comparison to experiments with steel and Dural rails (see Figs. 11 and 12 of [2]) shows that the highest $B$-field is concentrated at the edge of the copper rails. It agrees with theory that the diffusion time increases with increasing conductivity hindering the $B$-field to expand into the conductor [7]. The $B$-field strength strongly depends on the distance $\Delta x$.

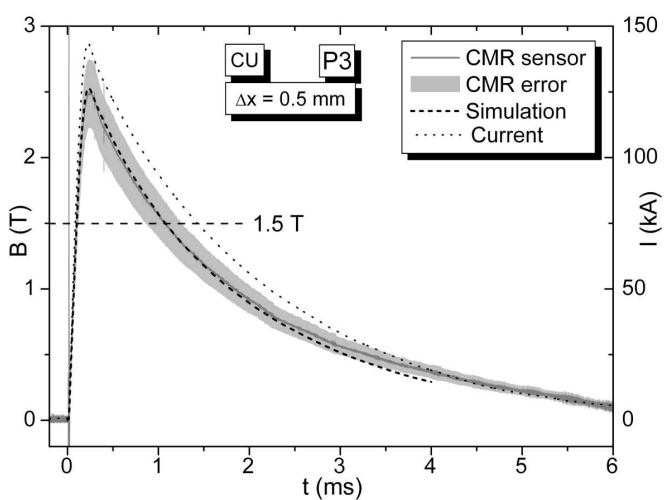

Fig. 2. Simulated and measured ( \pm error) $B$-field pulse and total current.
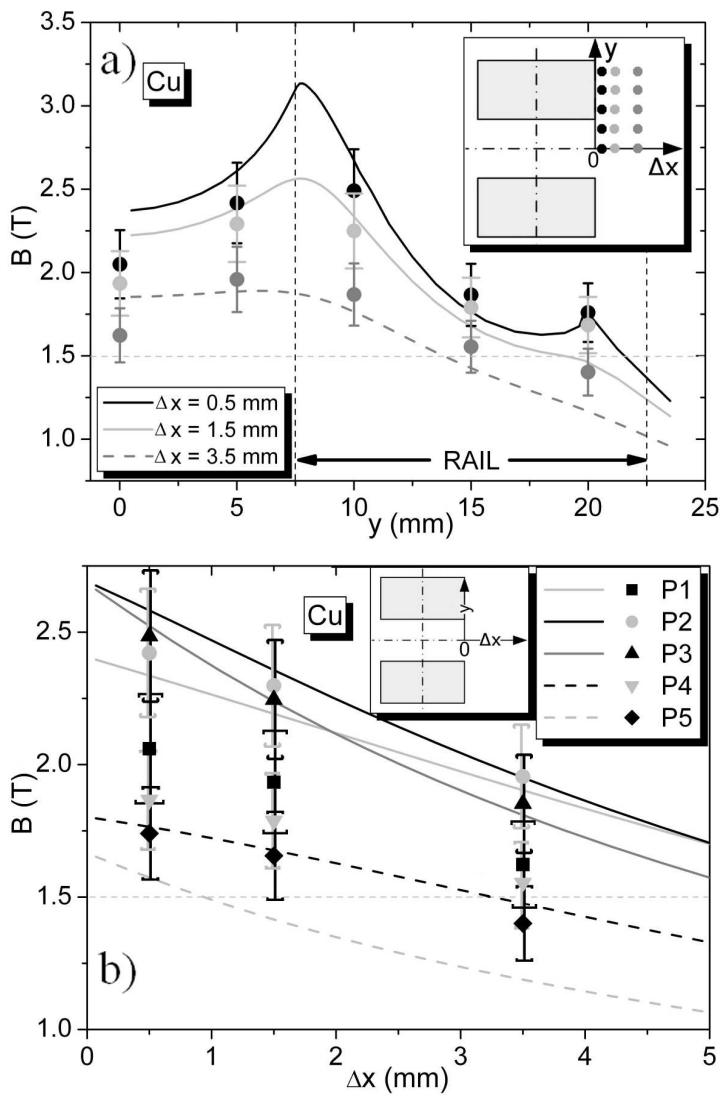

Fig. 3. Measured (points) and simulated (lines) $B$-field depending on $y(\mathrm{a})$ and on $\Delta x(\mathrm{~b}) . t=0.25 \mathrm{~ms}$.

For a dynamic experiment Fig. 4 shows the signal of the magnetic field sensors when the projectile passes the sensor position at $3.5 \mathrm{~ms}$ with a velocity scantly exceeding $1200 \mathrm{~m} / \mathrm{s}$. Focussing on the signals wave forms, the influence of the current distribution in the railgun can be seen: Note the differences of the curves at $3.55 \mathrm{~ms}$. With increasing distance to the inner rail surface the variation of the $B$-field with time becomes less pronounced resulting in a flattening of the measured curve. The reason is 


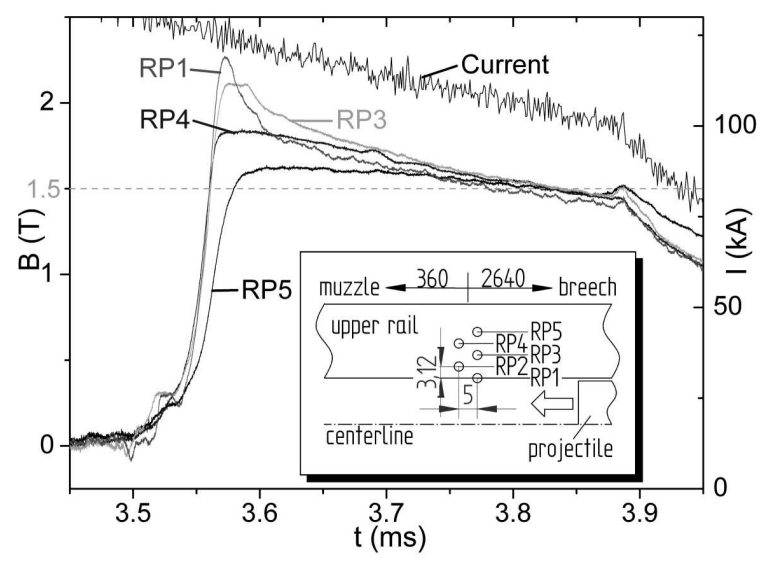

Fig. 4. $B$-field wave forms at a dynamic railgun experiment. All dimensions in $\mathrm{mm}$.

seen in a combination of the current concentration at the inner surface due to the skin effect and the passing short circuit of the projectile.

\section{Conclusions}

A new CMR- $B$-scalar sensor generation enables the detection of current concentration directly at the railgun rail surface during static and dynamic experiments. Magnetic field distributions were measured with projectile velocities exceeding $1200 \mathrm{~m} / \mathrm{s}$. Our investigations show that CMR- $B$-Scalar sensors are well suited as a diagnostic tool for investigations of transient strong magnetic fields and hence the current distributions in railguns.

\section{References}

[1] M. Schneider, R. Schneider, V. Stankevic, S. Balevicius, N. Zurauskiene, IEEE Trans. Magn. 43, 370 (2007).

[2] M. Schneider, O. Liebfried, V. Stankevic, S. Balevicius, N. Zurauskiene, IEEE Trans. Magn. 45, 430 (2009).

[3] M. Schneider, R. Schneider, IEEE Trans. Magn. 43, 186 (2007).

[4] D. Roger, H. Lai, IEEE Trans. Magn. 37, 135 (2001).

[5] M. Schneider, M. Woetzel, W. Wenning, D. Walch, IEEE Trans. Magn. 45, 442 (2009).

[6] M. Ziese, S.P. Sena, J. Phys., Condens. Matter 10, 2727 (1998).

[7] H.E. Knoepfel, Magnetic Fields: A Comprehensive Theoretical Treatise for Practical Use, Wiley, New York 2000. 